\title{
Erlösung aus dem Jammertal
}

«Transmodulationsblockade» nennt sich ein Verfahren, mit dem ein russischer Arzt namens Dr. Michael Zobin Opiatabhängige nachhaltig von ihrer Sucht befreien will. Wenn Ihnen diese Methode und ihr «Erfinder» trotz regelmässiger Lektüre wissenschaftlicher Fachjournale nichts sagen, brauchen Sie Ihre Fortbildungsgewohnheiten vorerst nicht zu hinterfragen: Gemäss den Recherchen von Ruedi Stohler und Andreas Moldovanyi, zweier ausgewiesener Spezialisten im Bereich Abhängigkeitserkrankungen, finden sich zumindest in der allgemeinzugänglichen Fachliteratur keine Arbeiten von Dr. Zobin. In Patientenkreisen sowie bei deren Angehörigen sind der russische Arzt und seine Methode dagegen sehr wohl ein Thema. Nachdem auch Therapeuten zunehmend mit Anfragen zur Transmodulationsblockade konfrontiert werden, sahen sich Stohler und Moldovanyi veranlasst, die verfügbaren Informationen in dieser Ausgabe der SÄZ in einem Artikel zusammenzufassen und zu kommentieren (ab Seite 610).

Angesichts der Resultate ihrer Nachforschungen erstaunt es nicht, dass aus fachärztlicher Sicht dringend von der «Wunderkur» abzuraten ist. Die Wahrscheinlichkeit, dass die undurchsichtige Methode hält, was sie verspricht, muss realistischerweise als verschwindend klein beurteilt werden - die Erklärungen auf der Homepage von «Doctor Zobin's Medical Centre - Moscow» lassen keine seriöse Beurteilung zu (sollte die Methode allen Unwahrscheinlichkeiten zum Trotz doch funktionieren, müsste man sich bei Dr. Zobin entschuldigen, könnte ihm aber den Vorwurf nicht ersparen, die Skepsis durch seine [Nicht-]Kommunikation geradezu erzwungen zu haben).

Fatal ist, dass bei Personen mit einer Abhängigkeitserkrankung durch Heilsversprechungen Zobinscher Art einmal mehr jene Mechanismen aktiviert werden, die ihnen im Verlauf der Suchtkarriere immer wieder zum Verhängnis geworden sind. Nicht zufälligerweise werden die Erwartungen an die «Kur» häufig unter Rückgriff auf eine religiös gefärbte Terminologie umschrieben. Man verspricht sich die Erlösung von der Sucht, erlebt eine Wiedergeburt, erhält ein neues Leben geschenkt. Und das alles - soweit es den Part in Moskau betrifft - ohne Anstrengung, lediglich dank einer Spritze (allerdings zu einem erklecklichen Preis und nach selbständig durchgeführtem körperlichem Entzug). Hatten wir dieses Versprechen nicht schon mal? Innerer Friede, Glücksgefühle, Erlösung von der Mühsal dieser Welt - alles dank einem «Schuss», ganz ohne Anstrengung.

Fachwissen hin oder her: Auch der gesunde Menschenverstand sträubt sich dagegen, den Heilsversprechungen aus Moskau zu trauen. Auf welchem Gebiet menschlicher Betätigung gibt es eine Methode, die ohne Anstrengung und ohne unerwünschte Begleiterscheinungen zum gewünschten Ziel führt? Wie wäre es zu erklären, dass ein relativ risikoarmes Verfahren mit einer traumhaften Erfolgsquote sich in über 10 Jahren nicht flächenbrandartig über den gesamten Globus verbreitet hat? Welche Motive könnte ein menschenfreundlicher Forscher haben, seine bahnbrechende Entwicklung der Wissenschaftsgemeinde und sehr vielen Patienten vorzuenthalten? Wo bleiben die Pharmafirmen, die sich um das massgeschneiderte Neuropeptid reissen?

Und was ist mit den Beispielen erfolgreicher Behandlungen, die auf Dr. Zobins Homepage angeführt sind? Alles frei erfunden? Wohl kaum. «Würden Sie Zobins Methode anderen Süchtigen empfehlen?» wird ein ehemaliger Patient da gefragt. Seine Antwort ist aufschlussreich: «Wenn jemand wirklich bereit ist, aufzuhören ja. Es ist eine schwere Entscheidung. Etwas Endgültiges. Ich bin froh, es gemacht zu haben.» Wer im Drogenbereich professionelle Erfahrungen gesammelt hat, weiss: Es gibt glücklicherweise immer wieder Patientinnen und Patienten, die sich aus ihrer Abhängigkeit befreien können wenn Sie dazu bereit sind. Auch ohne Reise nach Moskau.

Bruno Kesseli 